\title{
Neue Formen der Partizipation und Entscheidungskompetenz
}

Andréa Belliger

Leitung Learning Services PHZ Luzern und Leitung MBA eHealth - Gesundheit digital

Referentin an den InfoSocietyDays
Lange Zeit drehte sich die eHealth-Diskussion in der Schweiz um die Effizienzsteigerung von Prozessen, die Senkung von Kosten und die Reduktion von Fehlern durch den Einsatz neuer Informations- und Kommunikationstechnologien. Doch nun kommt Bewegung in diese Diskussion, die im Titel des diesjährigen Swiss eHealth Forums in Bern ihren Ausdruck findet: consumer driven ehealth. Was leicht und elegant über die Lippen kommt, impliziert einen fundamentalen Wandel weg von der Technik hin zum Konsumenten und Patienten als Treiber von Gesundheitsinnovation.

\section{Am Anfang war das Web 2.0}

Dieser Perspektivenwechsel hat mit der Entwicklung des Internets zu tun. Das Web hat sich in den letzten fünf Jahren von einem Medium der Informationspublikation hin zu einer Kommunikationsplattform entwickelt. Diese Entwicklung wird als Web 2.0 bezeichnet. Facebook, YouTube, Plattformen wie xing, LinkedIn oder StudiVZ, Blogs, Wikis, Twitter und Tausende von andern, zumeist kostenlosen Applikationen gehören dazu. Aus einem Trend, der 2005 begonnen hat, ist eine Bewegung geworden. Das Interessante und Neue an dieser Entwicklung kommt in den Begriffen «Social Software» und «Soziale Netzwerke» zum Ausdruck. Treiber der Entwicklung ist - vernetzen sich, kommunizieren mit anderen Patienten und Ärzten in Portalen und virtuellen Sprechstunden, tauschen Gesundheitsdaten aus und beeinflussen damit Selbstdiagnose, Arztwahl, Medikation und Therapie. Dabei steht das «e» bei ePatient, nicht so sehr für «elektronisch», als vielmehr für «empowered»- befähigt, aktiv, kompetent.

ePatients sind nicht nur in der Altersklasse der NetGeneration, bei den 15-30-Jährigen, die im und mit dem Internet aufgewachsen sind, zu finden. $\mathrm{Zu}-$ nehmend gesellen sich die Silversurfers, Menschen ab 50, dazu. Die ePatients sind zu einer neuen Einflussgrösse auf dem Gesundheitsmarkt geworden und fordern vom Gesundheitssystem Kommunikation, Partizipation und Transparenz oder kurz: eHealth 2.0.

\section{Von Information zu Kommunikation}

Dass Gesundheitsinformationen zunehmend via Internet erschlossen werden, ist keine neue Sache.

Patienten sind aber nicht nur digital informiert, sondern vernetzt. Online Health Communities, auf denen sich Patienten organisieren, austauschen und moralisch unterstützen, weisen enorme Wachstumszahlen auf [1]. Der Umgang mit Gesundheit und Krankheit geschieht zunehmend in Sozialen Netzwerken.

\section{Das «e» bei ePatient steht nicht so sehr für «elektronisch», als vielmehr für «empowered» - befähigt.}

Korrespondenz:

Prof. Dr. Andréa Belliger Institut für Kommunikation \& Führung IKF Morgartenstrasse 7 CH-6003 Luzern

andrea.belliger@ikf.ch www.ikf.ch
Einige Institutionen im Gesundheitswesen haben wie der Name sagt - nicht eine technologische Innovation, sondern eine soziale Bewegung. Im Zentrum des Web 2.0 steht eine einfache, aber bestechende Philosophie - jene des Teilens, Mitteilens, des Interagierens und des Partizipierens. Wenn das Web 1.0 die Vernetzung von Dokumenten war, dann ist Web 2.0 die Vernetzung von Personen.

\section{Von ePatients, NetGen und Silversurfers}

Die Auswirkungen dieser Entwicklung sind auch im Gesundheitsbereich spürbar. Im Zuge der Web 2.0-Bewegung entsteht eine vernetzte Konsumenten- und Patientengeneration: die ePatients.

ePatients kommunizieren und informieren sich auf vielfältige Weise. Sie lesen und schreiben in Blogs, diese Tendenz erkannt und kommunizieren via Social Media mit den Patienten und ihren Angehörigen. Die amerikanische Mayo Clinic tut dies sehr erfolgreich via Blog, Podcast, Diskussionsforen, Videokanal auf iTunes und YouTube, Facebook und Twitter [2].

Auch Versicherungen und die Pharmazeutische Industrie sind in Sozialen Netzwerken aktiv. Viele Pharmafirmen nutzen Twitter als Medium zur Krisenkommunikation oder als Tweets über Konferenzen und Studien. Dieser Kommunikationskanal ist sehr fragil und funktioniert nur, solange valable Information ins Netzwerk gestellt, der Gedankenaustausch gefördert wird und ein Dialog z. B. mit Patientengruppen stattfindet. 


\section{Empowerment und Partizipation}

Die Zugänglichkeit von qualitativ hochwertiger Information verändert die Rollen im Gesundheitswesen. Das Wissen liegt nicht mehr einseitig bei den Healthcare Professionals. Das verändert das Verhältnis und macht die Grenzen zwischen Experten und Laien durchlässig. Laien und Patienten sehen sich zunehmend weniger als passive Empfänger von Gesundheitsdienstleitungen, sondern als aktive und selbst-

\section{Forderung nach Transparenz}

Partizipation und Entscheidungskompetenz von Patienten setzt Qualitätstransparenz und Vergleichbarkeit von Gesundheitsdienstleistungen voraus. Von zunehmender Bedeutung sind deshalb Online-Ratingsysteme von Spitälern, Arztpraxen und Kassen [3]. Diese erhöhen die Transparenz, begünstigen den Wettbewerb und involvieren die Konsumenten in die Qualitätssicherung.

\section{Patienten haben keine grundsätzlichen Ängste in Bezug auf die Digitalisierung ihrer medizinischen Daten.}

bestimmte Kommunikationspartner, als Initianten von Präventionsmassnahmen, Verantwortliche für Gesundheitsmonitoring und Manager von «home based care» - als befähigt, kompetent und «empowered». Diese Art der Partizipation geht weit über das persönliche Gesundheitsmanagement hinaus. Das Potential der Beteiligung von Patienten an Innovation und gemeinsamer Wertschöpfung im Sinne einer «value co-creation» wird durch Social Media zunehmend ersichtlich.

\footnotetext{
14. InfoSocietyDays

vom 7. bis 11. März 2011 in Bern

Kongress für ICT-Anwendungen in der Informationsgesellschaft

Über 1200 Interessierte aus Wirtschaft, Verwaltung und Gesundheitswesen nehmen jedes Jahr an den InfoSocietyDays in Bern (BEA expo) teil. Der Kongress für Anwendungen der Informations- und Kommunikationstechnologien behandelt mit dem Swiss eEconomy Forum, dem Swiss eGovernment Forum und dem Swiss eHealth Forum drei wichtige Kernthemen der Informationsgesellschaft. Der Fokus liegt auf Einsatz und Nutzen der Informations- und Kommunikationstechnologien.

Weitere Informationen unter www.infosociety days.ch

Die Schweizerische Ärztezeitung ist Medienpartner der InfoSocietyDays.
}

Transparenz ist aber nicht nur bei den Dienstleistungen gefordert, sondern auch im Umgang mit der persönlichen Information der Kundinnen und Kunden. Patienten - so zeigte z. B. eine Studie von TA Suisse 2007 - haben keine grundsätzlichen Ängste in Bezug auf die Digitalisierung ihrer medizinischen Daten, sie fordern aber berechtigterweise die volle Kontrolle und Verwaltung der eigenen Daten und wollen eigenständig je nach Verwendung über den Datenzugang entscheiden.

\section{Ausblick}

Die soziale Bewegung des Web 2.0 hat das Gesundheitssystem erreicht. Die Tragweite dieser Umgestaltung lässt sich nur schwer prognostizieren. Health 2.0 und die Forderungen der ePatients nach mehr Kommunikation, Partizipation und Transparenz bergen ein grosses Potential für die Gestaltung und Weiterentwicklung unseres Gesundheitssystems [4].

\section{Literatur}

1 Z.B. CureTogether, PatientsLikeMe oder DailyStrenght.

2 podcasts.mayoclinic.org; newsblog.mayoclinic.org; sharing.mayoclinic.org

3 RateMDs.com, CompareyourCare.com, medicosearch.ch, docinsider.de

4 Das Thema eHealth 2.0 wird im Rahmen des neuen Weiterbildungsstudienganges CAS ehealth - Gesundheit digital (www.master-ehealth.ch) interdisziplinär diskutiert. 